\title{
Parallel Computing Efficiency Analysis for Electromagnetic Transient Optimization Decoupling Method
}

\author{
Zheng Weijie ${ }^{1, a}$, Xu Dechao ${ }^{1}$, Zhang Xing ${ }^{1}, X$ Sh Shen $^{1}$,Wang Xiangxu ${ }^{1}$ \\ ${ }^{1}$ China Electric Power Research Institute,Power system department, Haidian District of Beijing, \\ 100192, China \\ aemail: dianlidianzi_@163.com
}

Keywords: Optimal Decoupling, Parallel Simulation, Real-Time Simulation

\begin{abstract}
This paper mainly researches automatically optimal decoupling technology for electromagnetic transient. After making clear advantages and disadvantages and selection principles of long transmission line decoupling algorithm and node-splitting algorithm, it analyzes the influence of computing time of different elements, decoupling borders, communication between subnets, IO calls on simulation time and parallel computing efficiency. It obtains formulas of subnet execution time for different parallel simulation algorithm. It analyzed automatically optimal decoupling algorithm for electromagnetic transient in real-time simulation step size constraint which is suitable for mixing-step simulation and develops the algorithm based on ADPSS. Finally, it verifies the correctness of the algorithm through practical grid and achieves real-time simulation of the grid.
\end{abstract}

\section{Introduction}

ADPSS electromagnetic transient calculation program can operate network segmentation both in bus node and long transmission line[1-5], this paper analyzed electromagnetic transient automatic optimization decoupling method applicable to hybrid step simulation, and realized the algorithm on ADPSS development based on long transmission line decoupling network parallel algorithm and node splitting network partition parallel algorithm[1-6]. Because in the same situation the network division long transmission line use shorter calculation time, higher efficiency[6-9], so under the condition of a long transmission line in the system, long transmission line decoupling has the priority[10-15].

\section{Automatic Decoupling Optimization Algorithm For Parallel Computing}

Network partition and parallel computation, whose essence is allocating the original commitment by a machine of computing tasks into multiple machines to complete simultaneously, which did not reduce the total amount of computation, but increased the communication and coordination work between each machine[6-11]. If the execution time of the system is Ts, and the execution time of the parallel computation of $\mathrm{N}$ sub-system is TP1[1-6]:

$$
T_{p 1}=\frac{T_{s}}{N}+T_{c o m 1}+T_{c a l}+T_{w 1}
$$

When the long transmission line decoupling method is used, the execution time of the parallel computation of the $\mathrm{N}$ sub network is Tp2[1-6]:

$$
T_{p 2}=\frac{T_{s}}{N}+T_{\text {com2 }}+T_{w 2}
$$

$T_{\text {com1 }} 、 T_{\text {com } 2}$ are parallel communication time, mainly depends on the selected communication network speed; $T_{w 1} 、 T_{w 2}$ is synchronization waiting time between subnet, the network is more uniform (i.e.: each subnet size are almost consistent), $T_{w 1} 、 T_{w 2}$ is smaller; $T_{\text {cal }}$ is serial sections of the computing time in node splitting method parallel computing, which was used to calculate net contact current), regardless of network size and the number of subnets, but it is proportional to the 
number of network boundary bus.

As a given the scale of the network, before splitting the network, the realization of real-time simulation network number is unknown, so the automatic optimization decoupling algorithm in calculating the execution time of each sub network, need to correspondingly improve function (1) and (2). Moreover, in order to automatically decouple large-scale power grid system into uniform scale subnets, electromagnetic transient automatic optimization decoupling algorithm also need considering effects of different element calculation, uncoupled boundary, inter subnet communication and IO call to simulation time and parallel computational efficiency.

In order to consider components of different amount of calculation, uncoupled boundary of simulation time and parallel computational efficiency, the power system electromagnetic transient components for Mach、RLC、Trans、Nonliner、Vs、Is、Switch 、TO、 TI types, respectively, on behalf of the generator component, RLC elements, elements of the transformer, nonlinear components, voltage source element, current source components, switch components, physical output interface, physical interface input. The calculation time of each element is using $T_{M a c h} 、 T_{R L C}$ 、 $T_{\text {Trans }} 、 T_{\text {Nonliner }} 、 T_{V S} 、 T_{I S} 、 T_{\text {Switch }} 、 T_{T O} 、 T_{T I}$ respectively[1-6]. The total number of nodes is NB, divided the subnet number is $N$, various components number in subnet $\mathrm{K}$ is $N_{\text {Machk }} 、 N_{R L C k} 、 N_{\text {Transk }} 、 N_{\text {Nonlinerk }}$ 、 $N_{\text {Vsk }} 、 N_{\text {Isk }} 、 N_{\text {Switchk }} 、 N_{\text {TOk }} 、 N_{\text {TIk }}$ considering different element calculation, uncoupled boundary, net communication and IO call effect, using the node splitting method for network partition and parallel, subnet K execution time[1-9]:

$$
\begin{aligned}
& T_{p 1}=N_{\text {mach }_{-} k} T_{\text {mach } \_k}+N_{R L C_{-} k} T_{R L C_{-} k}+N_{\text {Trans }_{-} k} T_{\text {Trans } \_k}+N_{\text {Nonliner }_{-} k} T_{\text {Nonliner } \_k} \\
& +N_{V s_{-} k} T_{V s_{-} k}+N_{\text {Is } \_k} T_{I s_{-} k}+N_{\text {Switch }_{-}} T_{S_{\text {Switch } \_}}+N_{T_{O_{-} k}} T_{T O_{-} k}+N_{T I_{-} k} T_{T I_{-} k} \\
& +T_{\text {com } 1}+T_{I O 1}+T_{\text {cal }}
\end{aligned}
$$

When the long transmission line decoupling method is used, the execution time of the sub network $\mathrm{k}$ is[1-15].:

$$
\begin{aligned}
T_{p 2} & =N_{\text {mach } \_k} T_{\text {mach } \_k}+N_{R L C_{-} k} T_{R L C_{-} k}+N_{\text {Trans } \_k} T_{\text {Trans } \_}+N_{\text {Nonliner }_{-} k} T_{\text {Nonliner } \_k} \\
& +N_{V_{s_{-}} k} T_{V s_{-} k}+N_{\text {Is } k} T_{I s_{-} k}+N_{\text {Switch }_{-} k} T_{\text {Switch }_{-} k}+N_{T_{O_{-} k}} T_{T O_{-} k}+N_{T I_{-} k} T_{T I_{-} k} \\
& +T_{\text {com } 1}+T_{I O 1}
\end{aligned}
$$

$T_{\text {com1 }} 、 T_{\text {com2 }}$ is the sub network communication time, $T_{I O 1} 、 T_{I O 2}$ is call time for the IO.

Each calculation time of the components, the time of the sub network communication time, IO call time, the processing time of single section are configured in the algorithm configuration file.

\section{Algorithm Validation}

In order to verify the correctness of the automatic optimization of electromagnetic transient decoupling algorithm, this paper selects the actual network simulation as verification, the network wiring diagram is as shown in Figure 1, the size of the network is as follows:

$>$ The 50 PARK equation model of synchronous generator (with regulator);

$>$ The 118 three-phase two winding common transformer;

$>$ The 218 lines (including PI type three-phase lumped parameter line and distributed parameter line)

$>$ The 129 series RLC three-phase load components;

$>$ The 6 switches 


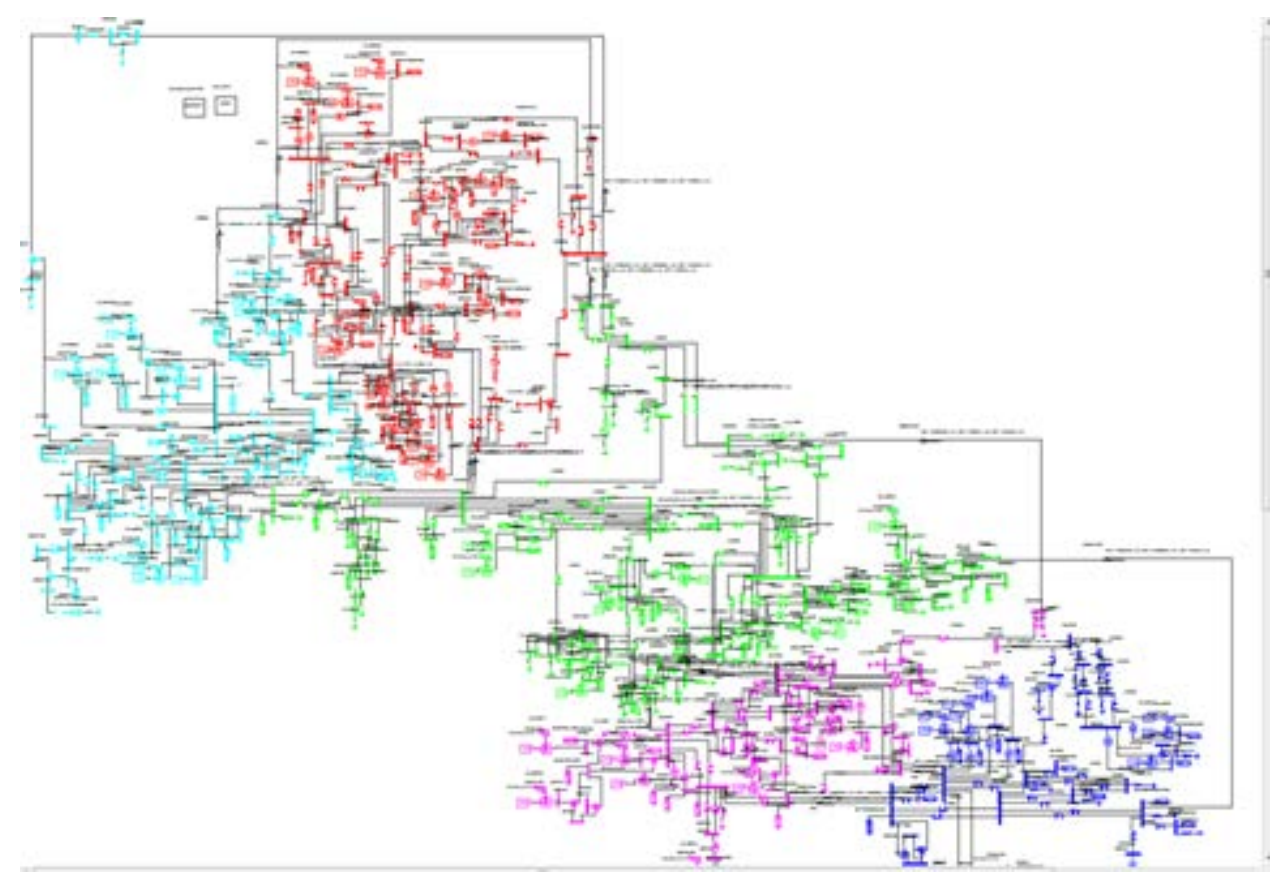

Figure 1 grid diagram

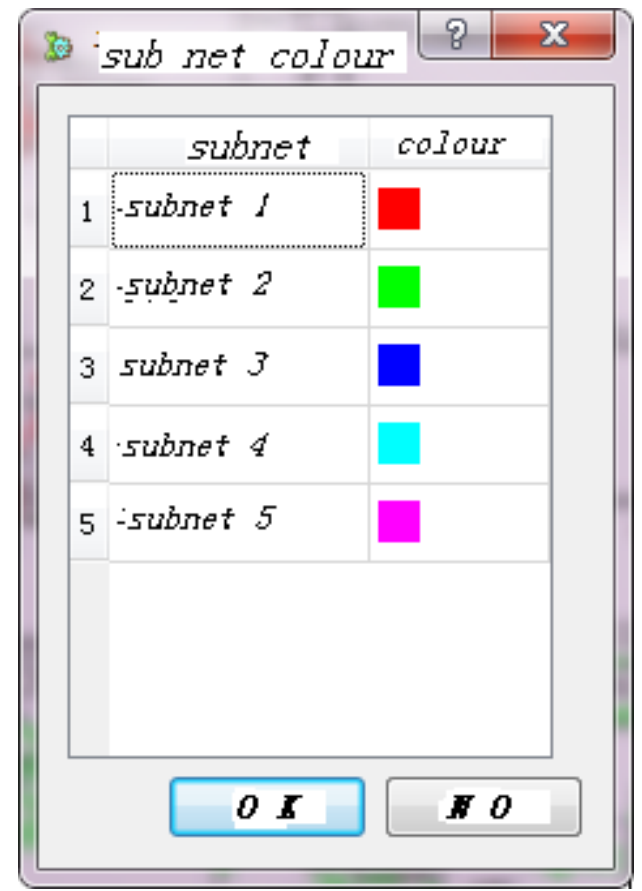

Figure 2 subnet division color

The electromagnetic transient simulation of the whole network can not meet the requirements of real time. With the purpose of real-time simulation, the automatic optimization of the network is divided into the sub-networks by the method of the automatic optimization of the electromagnetic transient. Figure 1 presents the ADPSS electromagnetic transient calculation program of actual power grid example implementation automatic network results figure, color in figure shows the sub network information for each element belonging to, each color element belongs to the subnet name, such as shown in Figure 2. In Figure 1, the contact line information is given in the sub networks case.

The following was used to verify the real-time and the correctness of the algorithm and network division.

In order to verify the correctness of automatic optimization decoupling algorithm, compares the simulation waveforms of whole network to network division scheme, figure 3 show a generator and a bus as an example, comparison of waveforms are given. 


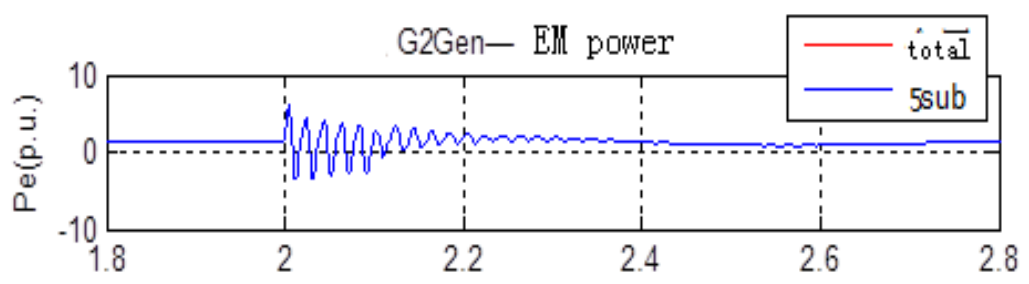

$t(s)$

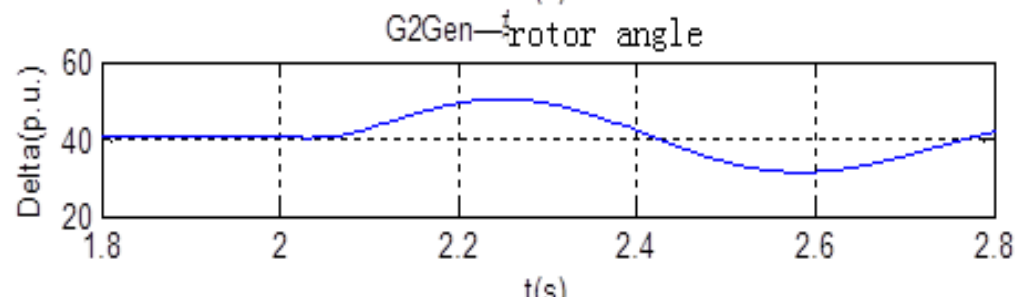

$\mathrm{t}(\mathrm{s})$

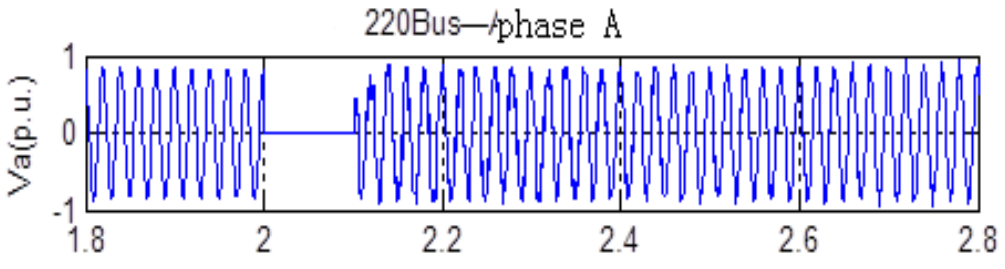

$t(s)$

Figure 3. Comparison of the output curve of the whole network and the sub network schemes

It can be seen that the output curves of the whole network and the network are consistent, which verifies the correctness of the automatic optimal decoupling algorithm.

In order to verify the numerical example of real-time (real time simulation criterion: the whole process of simulation, single step computation time is less than the simulation step), single step computation time in process simulation is in statistics, as shown in Figure 4 and table 1, among them, STmax is the largest single step computation time, STmin is the minimum computation time in one-step, STavg is average single step computation time, count is the number of steps which one-step calculation time is greater than simulation step.
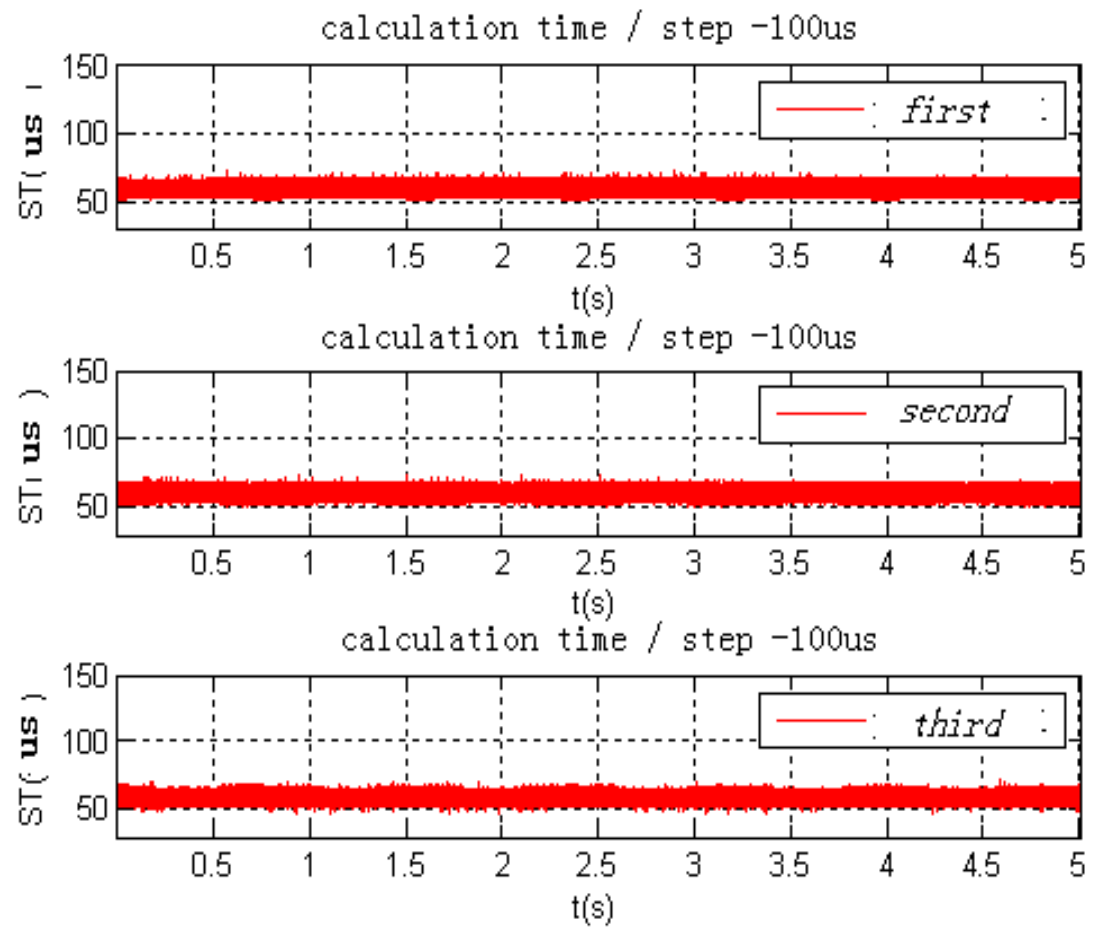

Figure 4. Single step calculation time of the three simulation (unit: us) 
Table 1. A single step calculation time table for distributed parameter line

\begin{tabular}{|c|c|c|c|c|}
\hline Single step calculation time ST(us) & first & second & third & average \\
\hline STmax(us) & 73.0180 & 73.6820 & 71.6660 & 72.7887 \\
\hline STmin(us) & 51.0050 & 50.8180 & 47.4780 & 49.7670 \\
\hline STavg(us) & 54.5127 & 54.0580 & 54.8384 & 54.4697 \\
\hline Count(us) & 0 & 0 & 0 & 0 \\
\hline
\end{tabular}

As we can be seen, the selected network in the case of automatic dividing into 5 sub networks can achieve real-time simulation of the network.

\section{Conclusion}

With the scale of electromagnetic transient simulation expanding, simulation calculation speed become slower, which increase the difficult to realize real-time simulation of the grid, the paper based on ADPSS carried out decoupling technology research of automatic optimization of electromagnetic transient, it mainly includes the following work:

In view of the parallel computing is the base for real-time simulation after automatic network optimization and decoupling, the paper introduces ADPSS existing two network partition and parallel algorithm, long distance line decoupling network partition parallel algorithm and node splitting network parallel algorithm, summarizing both the advantages and disadvantages of two parallel algorithm and selecting principle.

This paper analyses the parallel computing efficiency influencing factors, including different element computation decoupling boundary, subnet, IO call, it is concluded that the different sub network, subnet execution time calculation formula, hybrid simulation step in real-time simulation with time step constraint of electromagnetic transient automatic decoupling method is proposed, the core thought of the algorithm is that: the network according to certain decoupling relationship is divided into a lot of electrical sub networks, and then the electrical sub stations were combined into a certain number of subnets, the combination considering the effects of different element calculation, decoupling boundary, subnet, IO calls to the simulation time and parallel computing efficiency. Finally, the electromagnetic transient decoupling method which is suitable for the hybrid step simulation is realized based on the ADPSS development and implementation, the algorithm is proved to be correct by an example of actual power grid.

\section{References}

[1] Yue Chengyan, power system electromagnetic and electromechanical transient hybrid real-time simulation research[D], China Electric Power Research Institute PHD thesis, 2004

[2] S. Y. R. Hui, K. K. Fung, and C. Christopoulos. Decoupled simulation of DC-linked power electronic systems using transmission-line links[J]. IEEE Transactions on Power Electronics, 1994: 85-91.

[3] C.S. Chang , L.R.LU , F.S.WEN . Power system network partitioning using tabu search[J]. Electric Power systems Research, 1999: 55-61.

[4] ZHENG Weijie,Transient Control Algorithm and Strategy of Controllable Shunt Reactors Individual Phase Suppressing Overvoltage and Secondary Arc Current[J],Proceedings of the CSEE,2014.7.5, Vol.34 No.19,PP:3187-3193

[5] Irving $\mathrm{M}$. R . , Sterling M . J . H . Optimal network tearing using simulated annealing[J]. IEE Proceedings-Generation, Transmission and Distribution, 1990, 137 (1): 69-72. 
[6] Li Yalou, Large scale power system electromechanic transient real time simulation algorithm and software research[D],

[7] Zheng Wei-jie, Zhou Xiaoxin, Adaptive Tracking Algorithm for Magnetically Controlled Shunt Reactor Control, 2010 International Conference on Power System Technology[C], on October, 2010, in Hangzhou, China.pp:A-99 (EI Index).

[8] Gomez A, Franquelo L. G. A New Contribution to the Cluster Problem[J], IEEE Transactions on Circuits and Systems, 1987, 34(5): 546-552.

[9] De JONG E,de GRAAFF R,VAESSEN P,et al.European white book on real-time power hardware-in-the-loop testing. . 2011

[10]Snider L,Be X,langer J,et al.Today's power system simulation challenge:High-performance,scalable,upgradable and affordable COTS-based real-time digital simulators. 2010 Joint International Conference onPower Electronics Drives and Energy Systems (PEDES) . 2010

[11]ZHENG Weijie,Dynamic Difference Correction Tracing Control Algorithm for Bulk Capacity Nonlinear Elements of Power Systems[J],Proceedings of the CSEE,2014.5.5 , Vol.34 No.13,:2172-2177. Vol.34 No.19 Jul.5, 2014.

[12] Hairer E,N rsett S P,Wanner G.Solving ordinary differential equations i:nonstiff problems. . 2011

[13]PEKAREK S,WASYNCZUK O,WALTERS E,et al.An efficient multi-rate simulation technique for power-electronicbased systems. IEEE Power Engineering Society General Meeting . 2004

[14]Ren, Wei,Steurer, Michael,Baldwin, Thomas L.Improve the stability and the accuracy of power hardware-in-the-loop simulation by selecting appropriate interface algorithms. IEEE Transactions on Industry Applications . 2008

[15]Hui, S.Y.R.,Fung, K.K.,Christopoulos, C.Decoupled simulation of DC-linked power electronic systems using transmission-line links. IEEE Transactions on Power Electronics . 1994

First A. Author

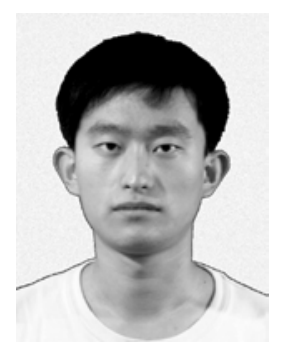

Zheng Weijie received the the Ph.D. degree fr China Electric Power Research Institute, Beiji China, in 2010, and M.SC. and B.Sc degree fr Shan-dong University, Jinan, China, in 2007 and 20 respectively. Currently, he is a senior engineer CEPRI. His main research interests are power mo simulation and control. dianlidianzi_@163.com 